Cite this: Nanoscale, 2014, 6, 3954

\title{
Facile colloidal coating of polystyrene nanospheres with tunable gold dendritic patches $\dagger$
}

\author{
Huixin Bao, ${ }^{\text {ad }}$ Timo Bihr, ${ }^{\text {bcd }}$ Ana-Sunčana Smith ${ }^{\text {bd }}$ and Robin N. Klupp Taylor ${ }^{\star a d}$
}

Patchy particles comprise regions of differing material or chemical functionality on otherwise isotropic cores. To meet the great potential of these anisotropic structures in a wide range of application fields, completely new approaches are sought for the scalable and tunable production of patchy particles, particularly those with nanoscale dimensions. In this paper the synthesis of patchy particles via a simple colloidal route is investigated. Using surfactant-free cationic polystyrene nanospheres as core particles, gold patches are produced through the in situ reduction of chloroauric acid with ascorbic acid. The fact that such nanostructured metal patches can be heterogeneously nucleated on polymer nanospheres is related to the electrostatic interaction between core and metal precursor. Furthermore, the lateral expansion of the gold patches over the polystyrene surface is facilitated by an excess of ascorbic acid. The morphology of the patches is highly dendritic and process-induced variations in the structure are related to gold surface mobility using Monte Carlo simulations based on the diffusion limited aggregation principle. Considering the $\mathrm{pH}$ dependent behaviour of ascorbic acid it is possible to predict the moiety which most likely adsorbs to the polymer surface and promotes gold surface diffusion. This enables the judicious adjustment of the $\mathrm{pH}$ to also obtain non-dendritic patches. On account of the plasmonic behaviour of gold, the patchy particles have morphology-dependent optical properties. The systematic development of the synthetic approach described here is expected to lay a foundation for the development of functional materials based on the self- or directed-assembly of nanoscale building blocks with anisotropic interactions and properties.

Received 1st August 2013

Accepted 4th November 2013

DOI: 10.1039/c3nr04016j

www.rsc.org/nanoscale

\section{Introduction}

The interest in so-called patchy particles has risen significantly in the last few years due to strong theoretical motivation in addition to their promising practical advantages. The growing status of the field is underlined by the numerous reviews published recently and covering patchy or Janus particles, the latter being a patchy particle with a single $50 \%$ coverage patch. ${ }^{1-6}$ The surface heterogeneity of patchy particles permits their use as building blocks for complex structures by self-assembly, ${ }^{7}$ and they have numerous possibilities as interfacial stabilizers, ${ }^{8}$ chemically or magnetically driven swimmers for drug delivery, ${ }^{9}$ and as sensor

Institute of Particle Technology, FAU Erlangen-Nürnberg, Cauerstr. 4, 91058 Erlangen, Germany. E-mail: robin.klupp.taylor@fau.de

${ }^{b}$ Institute of Theoretical Physics, FAU Erlangen-Nürnberg, Nägelsbachstr. 49b, 91052 Erlangen, Germany

${ }^{c}$ II. Institute of Theoretical Physics, University of Stuttgart, Pfaffenwaldring 57/III, 70550 Stuttgart, Germany

${ }^{d}$ Cluster of Excellence "Engineering of Advanced Materials", FAU Erlangen-Nürnberg, Nägelsbachstr. 49b, 91052 Erlangen, Germany

$\dagger$ Electronic supplementary information (ESI) available: Further details of the experimental conditions used in the work; SEM images showing the effect of reducing agent, ascorbic acid concentration, core particle concentration, chloroauric acid concentration, temperature and core particle type; zeta potential measurements of core particles. See DOI: 10.1039/c3nr04016j components. $^{\mathbf{1 0}}$ Furthermore, patchy particles comprising metallic patches ${ }^{\mathbf{1 1}}$ such as gold or silver on dielectric cores such as metal oxide, ${ }^{12-14}$ or polymer, ${ }^{15,16}$ have drawn much attention due to their promising optical properties. ${ }^{17,18}$ The synthesis of metallic patchy particles can be classified into two categories depending how the surface asymmetry is introduced: with and without interfacial templating. The immobilization of particles on a flat substrate followed by the sputtering of metal ${ }^{19}$ and the Pickering emulsion method ${ }^{20}$ are typical examples of patchy particle fabrication with interfacial templating. The advantage of these techniques is that the obtained particles have uniform morphology. However, serious drawbacks of the approach are lack of tunability (the formation of an arbitrary number of patches in defined locations) and prohibitive scalability.

Patchy particle fabrication without interfacial templating has so far received rather less attention. We recently reported a facile and versatile colloidal route for the synthesis of silver or silver-gold patches on non-functionalized silica particles in aqueous solution. ${ }^{\mathbf{2 1 , 2 2}}$ Our approach is based on the heterogeneous nucleation of silver directly onto the bare silica surface followed by surface diffusion dominated growth. Although protocols for metal coating of silica particles are already wellknown, these mainly rely on a preceding core functionalization step and usually lead to complete shell coatings or metal 
nanoparticles homogeneously distributed over the surface. Our novel approach relies on non-functionalized cores and makes use of low metal ion concentrations combined with carefully controlled reduction reactions. It was demonstrated that the patch morphology can be tuned from dense cup-like structures to dendritic patches simply by changing the reaction temperature or reactant addition rate. The strong affinity of the metal for the (amorphous) oxide surface observed in that work was unprecedented and led us to investigate whether such a simple synthetic process could be expanded to other materials systems. However, we found that when the silver precursor was replaced with chloroauric acid, no choice of reducing agent could result in the heterogeneous nucleation and growth of gold patches. This negative result is backed up by related literature, which also indicates that while functionalization of silica with amine groups enables some heterogeneous nucleation of gold to occur, there is no surface growth and instead the formation of surface-bound spheroidal nanoparticles. ${ }^{23}$ We propose the hypothesis that the relative sign of the electrostatic charge of the core particle and metallic precursor ions or complexes is a critical factor in the heterogeneous nucleation of metal patches as it permits the necessary surface supersaturation for nucleation to be attained. In other words, metals with positively charged precursor ions e.g. $\mathrm{Ag}^{+}$would nucleate on anionic cores whereas those with negatively charged ions e.g. $\left[\mathrm{Au}(\mathrm{OH})_{x} \mathrm{Cl}_{4-x}\right]^{-}$ would prefer cationic cores. Furthermore, we suggest that surface conformal growth relies on the presence of "sticky" interactions (e.g. hydrogen bonds) between at least one reactant and the core particle. This would increase the chance that components of the growing patch arrive predominantly at the patch edge (via surface diffusion) rather on the patch face, leading to patch thickening i.e. outwards into the bulk solution.

To investigate these hypotheses, we selected polystyrene nanospheres as core particles due to the fact that they can be synthesized with both strongly anionic and cationic character, avoiding the need for post-functionalization as in the case of silica particles. Having demonstrated that our previously reported one-pot approach for silver patch growth on silica could be successfully repeated for anionic polystyrene nanospheres (data not shown), we considered whether gold patches could likewise be formed on cationic polystyrene cores. This will be the main theme of the present work.

Besides aiming to demonstrate further generalization of the heterogeneous nucleation and growth approach for patchy particle synthesis, the work described herein adds to a growing interest on functional gold nanostructures fabricated on polystyrene core particles. Most of the related literature has focused on forming a homogeneous distribution of gold nanoparticles or even a complete gold nanoshell on PS spheres. In the former case, a popular approach has been to adsorb gold nanoparticles onto the core particle surface either by electrostatic interaction or by covalent bonding. ${ }^{\mathbf{2 4 2 5}}$ For instance, 2-aminoethanethiol hydrochloride was used to modify polystyrene particles so that it enabled the covalent binding of gold nanoparticles by thiol groups. ${ }^{26}$ In addition, inverting the surface charge via the Layerby-Layer technique and subsequently absorbing oppositely charged nanoparticles is another commonly utilized approach to fabricate this kind of composite material. ${ }^{27}$ The swelling and deswelling of PS microspheres in aqueous dispersion in response to tetrahydrofuran has also been demonstrated as a means to deposit preformed gold nanoparticles onto the core surface. ${ }^{28}$ In that work it was also showed in one micrograph that patchy coatings with a dendritic morphology could be obtained although the potential significance of this was apparently not recognized and the phenomenon not investigated further.

To produce a complete gold nanoshell from a nanoparticle "seeded" core, further deposition of metal via electroless plating leads to an increase in the size of the seed nanoparticles until a complete shell is formed. ${ }^{26}$ Another approach is "seedless" direct metallization, namely, the in situ reduction of a metallic precursor in the presence of the core particles leading to the deposition of metal on the surface..$^{29,30}$ Along these lines, the reduction of gold with aniline-swelled anionic PS microspheres led to the formation of large gold nanoparticles arrayed on the surface of the cores. ${ }^{31,32}$ Furthermore, Li et al. recently carried out the reduction of gold in the presence of cationic PS using a range of reducing agents, including ascorbic acid, as used in the present work. ${ }^{33}$ They claim that the obtained gold-spheroid decorated spheres formed via heterocoagulation of homogeneously nucleated gold nanoparticles and the core particles.

Jang et al. reported the direct metallization of silica spheres functionalized with an amine-rich multilayer using ascorbic acid as reductant and polyvinylpyrolidone (PVP) as surface active additive. ${ }^{12}$ Although the authors of that work were clearly aiming to produce complete gold nanoshells, an intermediate step in the growth was shown in one micrograph to be a partial coating of the core with a single large patch having a dendritic morphology. The authors attribute this morphology, which is rather analogous to the dendritic structures observed in our earlier work on silver patchy particles, to diffusion limited aggregation (DLA) of gold nuclei at the core's surface. Although following a similar approach to that described in the current work, the mechanism stated by Jang et al. raises questions regarding the exact contribution of the ascorbic acid and PVP to the dendritic patch growth.

In the current article we show that in fact cationic PS nanospheres can be coated with multiple gold patches using only ascorbic acid to reduce the auric ion. In particular, our work constitutes the first explicit study of a one pot approach to produce gold patchy particles in homogeneous colloidal solution i.e. without interfacial or emulsion templating. We also demonstrate, as with our earlier work on silver patch coatings of silica, that simple process variables can lead to morphological control over the patches formed. By means of systematic experimentation and supported by Monte Carlo simulation of diffusion limited aggregation we show that the dendritic morphology of the patch is particularly sensitive to the ascorbic acid concentration, the $\mathrm{pH}$ and the reaction temperature. Our work shows an important step in the establishment of a scalable chemical approach for the formation of anisotropic gold coatings directly onto nanospheres and reveals possibilities for tuning the patch morphology by controlling the surface interaction. The patchy particles show morphology dependant optical properties. Our approach, in comparison with almost all other studies into metal coating of non-metallic core particles may be highly suited for 
future work to produce large quantities of surface anisotropic building blocks for self-assembled functional materials.

\section{Methods}

\section{Synthesis of polystyrene nanospheres}

Cationic polystyrene (PS) nanospheres were synthesized under a surfactant-free condition as previously described. ${ }^{34}$ Briefly, the synthesis was carried out in a three-necked round bottom flask fitted with a condenser. A portion of $150 \mathrm{~mL}$ Millipore water was firstly bubbled with nitrogen for 1 hour at $40{ }^{\circ} \mathrm{C}$. The initiator, 2,2'-azobis(2-methylpropionamidine) dihydrochloride (97\%, $\mathrm{V}-50$, Sigma-Aldrich GmbH, Germany), $0.15 \mathrm{~g}$ dissolved in $1 \mathrm{~mL}$ water, was added, followed by the addition of a mixture of $68 \mathrm{~mL}$ styrene monomer (99\%, Merck KGaA, Germany) and $0.28 \mathrm{~mL}$ hexadecane (Sigma-Aldrich). The polymerization was carried out at $80{ }^{\circ} \mathrm{C}$ under nitrogen bleed for 24 hours. The particles were subsequently washed by centrifugation and re-dispersion in water. The concentration of the final PS nanospheres suspension was $160 \mathrm{mg} \mathrm{mL}^{-1}$. For the synthesis of gold patchy particles, a 10-fold diluted suspension was used. The mean and standard deviation particle diameters as determined by scanning electron microscopy (50 particles measured) were $256 \mathrm{~nm}$ and $48 \mathrm{~nm}$ respectively. The mean zeta-potential as determined by electrophoretic light scattering at neutral $\mathrm{pH}$ was $+32.7 \mathrm{mV}$. Due to the cationic character arising from the anchored amidinium groups provided by the initiator, the suspension remained stable over a long period and no further stabilizing agents were added.

\section{Synthesis of gold nanostructures on PS nanospheres}

Tables S1 and S2 in the ESI $\dagger$ provides an overview of the synthetic conditions for the samples described in this work. Gold nanostructure-decorated PS nanospheres were fabricated by reducing aqueous chloroauric acid (gold chloride trihydrate, 99.9\%, Sigma-Aldrich) with aqueous L-ascorbic acid (99\%, Carl Roth $\mathrm{GmbH}$, Germany) in the presence of the core particles. This reaction was initially performed in the order (1) PS dilution, (2) chloroauric acid addition, (3) ascorbic acid addition while later the order of addition of chloroauric and ascorbic acids was reversed i.e. the gold precursor was added to a stirred mixture of PS nanospheres and ascorbic acid. In all cases the three components had a total concentration in the reaction mixture of typically $16 \mu \mathrm{g} \mathrm{mL}^{-1}$ (PS), $0.15 \mathrm{mM}$ (chloroauric acid) and 0.01$100 \mathrm{mM}$ (ascorbic acid). A color-change corresponding to the formation of gold nanostructures was observed immediately following addition of the final reactant. Without further treatment, the optical extinction spectrum of the product suspension was obtained. Prior to microscopical characterization, the particles were separated from the mother solution by centrifugation (14 $000 \mathrm{rpm}$ for 5 minutes) and were re-dispersed in water.

\section{Synthesis of gold nanostructures on PS nanospheres with different functional groups and amine functionalized silica spheres}

To demonstrate the effect of using alternative cationic spheres in the gold patch coating reaction, amino $\left(-\mathrm{NH}_{2}\right)$ functionalized PS and silica spheres were used. A suspension of polystyrene particles terminated with amino groups was purchased from Micromod Particle Technology GmbH (Germany) and was used without further treatment. Silica spheres (Monospher M500, Merck KGaA, Germany) functionalized with amine groups were used to replace the polystyrene. The functionalization of silica with 3-aminopropyltriethoxysilane (99\%, Sigma-Aldrich) was carried out as detailed in the ESI. $\uparrow$ Table S3 in the ESI $\uparrow$ provides the details of the synthetic conditions for gold coating of the amino functionalized PS and silica spheres.

\section{Characterization}

The optical extinction spectra of the product dispersion were measured by using a Lambda 35 spectrophotometer (PerkinElmer GmbH, Germany). The washed product dispersion was dropped onto silicon substrates, allowed to evaporate and was examined using an ULTRA 55 field emission SEM (Carl Zeiss AG, Germany) operating at $10 \mathrm{kV}$. Transmission electron microscopy (TEM) in high resolution and diffraction contrast modes was carried out using a CM 300 Ultra Twin microscope (Philips/FEI Company, The Netherlands). TEM samples were prepared by dropping the washed product dispersion on a copper grid supported carbon film. The size distribution and zeta potential of the PS nanospheres were measured using a Zetasizer instrument (Nano ZS, Malvern Instruments $\mathrm{GmbH}$, Germany). The particles produced in this work were analyzed by X-ray diffraction (XRD) using a D8 Advance instrument (Bruker AXS GmbH, Germany) with $\mathrm{CuK} \alpha$ radiation $(\lambda=0.15405 \mathrm{~nm})$. The diffraction experiments were conducted at $2 \theta$ values ranging from $10^{\circ}$ to $80^{\circ}$ with a step size of $0.141^{\circ}$, a step time of $2 \mathrm{~s}$, an applied potential of $30 \mathrm{kV}$ and $40 \mathrm{~mA}$ current. The sample for measurement was prepared by drop casting the particles suspension onto the holders which comprise low background sample cups with a vicinal (911) Si crystal of $25 \mathrm{~mm}$ diameter (Bruker AXS GmbH).

\section{DLA simulations}

We perform Monte Carlo simulations of diffusion limited aggregation $^{35}$ with a finite sticking probability $s .{ }^{36}$ Thereby it is well accepted that lowering the sticking probability increases the density of a growing dendrite, ${ }^{37}$ which is related to the survival time of a free tracer when in contact with the dendrite. To account for the finite nature of surface diffusion processes occurring on the PS nanospheres, the simulations were executed on a quadratic square grid of the size $1024 \times 1024$ sites and using periodic boundary conditions. Thereby curvature effects are not taken into account as they are considered to be small. ${ }^{38}$ Furthermore, since the absorption of gold onto the nanospheres is assumed to be fast compared to the growth of the cluster, all tracers (gold atoms) were placed randomly at time $t=0$ on the grid (the PS colloid) to meet an initial density of choice. Each simulation step propagates the system for $\delta t$, when each free tracer performs a self-avoiding random walk with a diffusion constant $D=a^{2} / 4 \delta t$, with $a$ being the lattice constant. The domain is nucleated at the beginning of the simulation by a single immobilized tracer particle, placed in the 
middle of the lattice. A free particle positioned on a neighbouring site of the domain may attach to the domain with a DLA sticking probability $s(0 \leq s \leq 1)$, which is for the current purposes defined as

$$
s=1-\mathrm{e}^{-k_{\mathrm{on}} \delta t} .
$$

Here $k_{\text {on }}$ should be interpreted as the binding probability of two gold atoms in the domain. Binding to a growing domain depletes the reservoir of diffusing tracers, which ultimately limits the growth to a finite size agglomerate. For small $k_{\mathrm{on}}$

$$
s \approx \frac{k_{\mathrm{on}} a^{2}}{4 D}
$$

Typically, as the sticking coefficient vanishes, the fractal dimension becomes close to the spatial dimension - close to 2 in the current case.

For presentation purposes, the clusters obtained in simulations are shown with the same resolution as the experimental electron microscopy images.

\section{Results and discussion}

\section{Synthesis of gold patches on PS particles}

The starting point for the present work was motivated by our previous finding that when anionic silica nanospheres were dispersed in a dilute solution of silver nitrate and a suitable reducing agent added, the heterogeneous nucleation and growth of surface-conformal silver patches occurred. ${ }^{21}$ We test our hypothesis, that the electrostatic interaction between core particle and metal precursor was the main factor determining the success of heterogeneous nucleation, by exploring whether gold patches can be formed on cationic spheres from the negatively charged chloroauric ion. We therefore dispersed an aliquot $(20 \mu \mathrm{L})$ of $16 \mathrm{mg} \mathrm{mL}^{-1}$ cationic PS nanospheres in $5 \mathrm{~mL}$ of $0.15 \mathrm{mM} \mathrm{HAuCl}_{4}$ aqueous solution. To this dispersion, small aliquots $(<50 \mu \mathrm{L})$ of ascorbic acid with concentrations of either $0.5 \mathrm{M}$ or $0.1 \mathrm{M}$ were added rapidly during vigorous stirring. Upon the addition of ascorbic acid, the colour of the suspension immediately changed to greenish-blue for larger ascorbic acid amounts and dark purple for smaller. SEM images of four samples prepared in this way are shown in Fig. 1. It can be seen
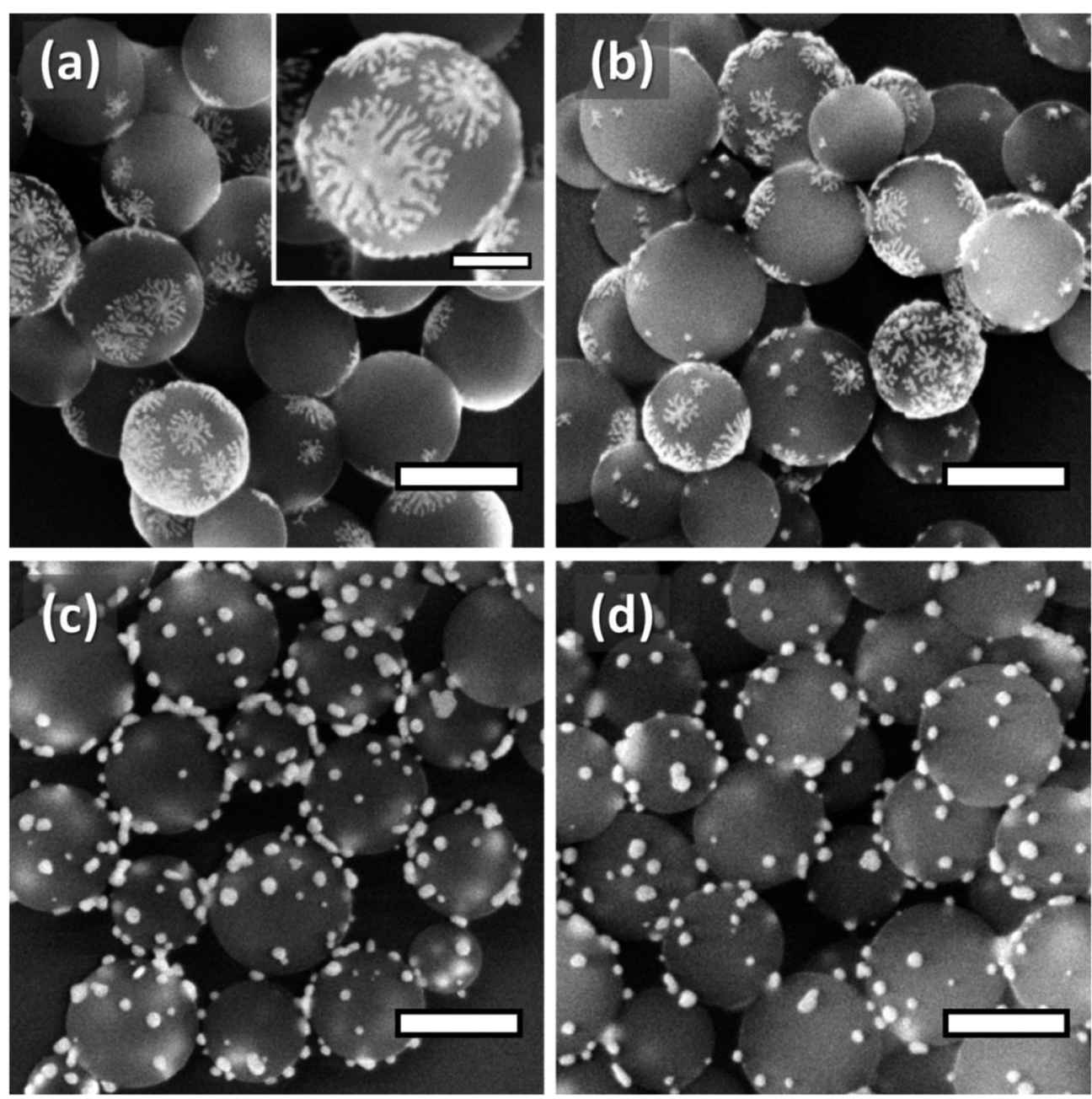

Fig. 1 SEM images of gold nanostructure-decorated cores formed by adding different amounts of ascorbic acid into a mixture of chloroauric acid and cationic PS nanospheres. The ascorbic acid amounts added were (a) $2.5 \times 10^{-5} \mathrm{~mol}$, (b) $2.5 \times 10^{-6} \mathrm{~mol}$, (c) $1.25 \times 10^{-6} \mathrm{~mol}$, (d) $6.25 \times$ $10^{-7} \mathrm{~mol}$. Scalebars correspond to $250 \mathrm{~nm}$ (main micrographs) and $100 \mathrm{~nm}$ (inset). 
that for larger additions of ascorbic acid (Fig. 1a and b) the polystyrene nanospheres are coated by multiple gold patches with a dendritic morphology. While all core particles possess a patch it is clear that the number of patches per core is somewhat distributed. In particular, for the lower amount of ascorbic acid (Fig. 1b), both small dendritic patches and spheroidal particles, possibly with small surface protrusions are seen. For even smaller ascorbic acid amounts (Fig. 1c and d) no dendritic patches are observed, but rather, the nanospheres are decorated by spheroidal and slightly elongated gold islands. As is already well known, the reduction of the auric ion by ascorbic acid is stoichiometric at a molar ratio of ascorbic acid to gold $\left(\mathrm{AA}: \mathrm{Au}^{3+}\right.$ ) of $1.5 .^{39}$ Given that the sample corresponding to Fig. $1 \mathrm{c}$ was formed at an $\mathrm{AA}: \mathrm{Au}^{3+}$ ratio of 1.67 it is clear that the dendritic patch morphology results from an excess of ascorbic acid.

This initial result indicates both similarities and differences with our earlier work on silver patch formation. In both cases heterogeneous nucleation occurs, though our experience shows that a high yield (fraction of cores possessing a patch) is less easily achieved in the case of silver-silica. ${ }^{21}$ In the present case, the widespread heterogeneous nucleation may result from the fact that both the gold precursor, $\left[\mathrm{Au}(\mathrm{OH})_{x} \mathrm{Cl}_{4-x}\right]^{-}$and the ascorbic acid could be expected to adsorb to the core particles, further raising the probability of nucleation. Another similarity between the two systems is the surface conformal growth of a metal on a curved non-metallic surface. In the case of silversilica fairly continuous patches of metal were produced although these could become somewhat dendritic at higher reaction temperatures. ${ }^{21}$ In contrast, the dendritic gold patches formed in the present case are rather finer suggesting that surface diffusion of gold on the PS nanospheres is slower. Furthermore, we did not observe a dendrite-to-nanospheroid transition in the earlier case of silver patchy particle formation. This suggests that there is a minimum concentration of ascorbic acid necessary to induce adsorption of gold that weakly binds to the surface of the core particle and undergoes surface diffusion.

Our present result is also in contrast to the findings of several other groups who reduced gold precursors in the presence of core particles with an opposite valence. Li et al. used cationic PS particles synthesized with azoisobutyronitrile initiator and stabilized with polyvinylpyrrolidone (PVP). ${ }^{33}$ Using various reducing agents and chloroauric acid, the authors obtained only gold-spheroid decorated particles, a result attributed to a heterocoagulation process but which could also have been explained by heterogeneous nucleation. It should be noted that in our case the gold concentration used was 3.33 times lower and we cover a wider range of $\mathrm{AA}: \mathrm{Au}^{3+}$ ratios (between 3 times lower and 10 times higher). This, together with the fact that our system is PVP free underlines the profound effect of ascorbic acid on the patch growth. Further earlier work reported that cationic polyelectrolyte grafted polystyrene particles can interact with gold ions $\left(\mathrm{AuCl}_{4}{ }^{-}\right)$electrostatically and result in a homogeneous distribution of gold nanoparticles over the surface when using sodium borohydride as reducing agent. $^{\mathbf{4 0 , 4 1}}$ Finally, the work of Lee et al. showed the direct deposition of gold on anionic polystyrene particles by using a cationic gold precursor to create the stronger interaction between the surface. Once again, however, a homogenous distribution of gold nanoparticles on the surface was obtained. ${ }^{42}$ It is therefore clear that while some structures reported in the literature are quite similar to what we observed for lower concentration of ascorbic acid (Fig. 1c and d) gold morphologies corresponding to the clear patchy structures on multiple particles (Fig. 1a and b) have never been reported before.

The relevance of ascorbic acid for producing surface conformal patches was also made clear by our own attempts to use alternative reducing agents. Fig. S1† shows that when ascorbic acid is replaced with formaldehyde, sodium borohydride, sodium citrate or hydroxylamine, heterogeneous nucleation occurs but no evidence of surface conformal growth can be seen. In the case of formaldehyde, large flower-like structures protrude from the PS cores. For all other reducing agents various sized spheroidal gold nanoparticles were formed on the surface as was previously reported. ${ }^{32}$ Extremely small surfacebound nanoparticles were formed with the strongest reducing agent (sodium borohydride). For trisodium citrate, along with gold nanospheroids, rod- and prism-like structures are observed. These do not appear to be affected by the reducing agent concentration, however. Thus, while it is clear that the heterogeneous nucleation of gold on cationic PS nanospheres can be achieved using other reducing agents, none of them are able to provide the necessary interfacial conditions to enable surface conformal growth.

\section{Effect of ascorbic acid concentration on patchy particle morphology and optical properties}

To explore in more depth the influence of the ascorbic acid we reversed the order of addition of the reactants. In other words, in a series of nine syntheses we mixed a constant amount of PS nanospheres $(20 \mu \mathrm{L})$ with $5 \mathrm{~mL}$ of ascorbic acid solution having a concentration in the range $0.05 \mathrm{mM}$ to $100 \mathrm{mM}$. Following this, $30 \mu \mathrm{L}$ of a $25 \mathrm{mM}$ chloroauric acid solution was injected. This reaction series corresponded to a variation of the $\mathrm{AA}: \mathrm{Au}^{3+}$ ratio from 0.3 to 667 . We intended that the order reversal should eliminate any dynamic effects relating to the ascorbic acid adsorption at the same time as the gold reduction is proceeding. Fig. 2 show SEM images of typical products from the series (larger area micrographs are included in the ESI, Fig. $\mathbf{S} 2 \dagger)$. As in the case where the ascorbic acid was added after the chloroauric acid (Fig. 1) we observe a clear transition in morphology. At very large ascorbic acid concentrations (10$100 \mathrm{mM}$, corresponding to $\mathrm{AA}: \mathrm{Au}^{3+}$ ratios of $\left.67-667\right)$ welldefined dendritic patches with continuous branches are seen along with a small number of loosely bound spheroidal nanoparticles apparently resulting from homogeneous nucleation. At somewhat lower concentrations $(2.5-5 \mathrm{mM}$, corresponding to $\mathrm{AA}: \mathrm{Au}^{3+}$ ratios of 17-33) dendritic patches are also seen, albeit with apparently coarser dendrites and displaying a high degree of pearling at the tips of the dendrites. The latter is most likely caused by thinning of the dendrite due to the finiteness of the 

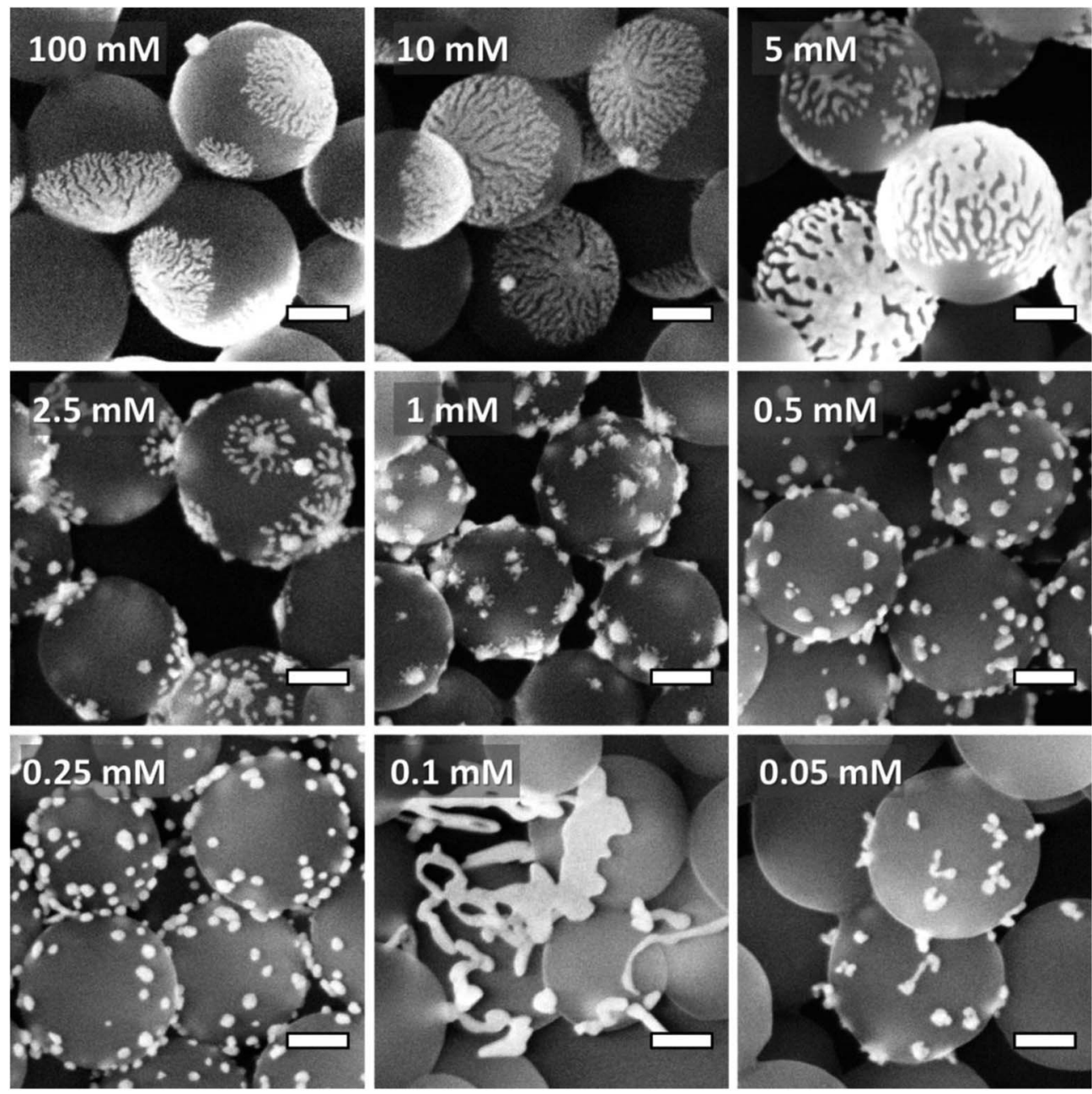

Fig. 2 SEM images of gold nanostructure decorated cores formed by mixing the same amount of cationic PS nanospheres with different concentrations of ascorbic acid (see image label) followed by addition of the same amount of chloroauric acid. Scalebars correspond to $100 \mathrm{~nm}$.

reservoir of diffusing gold atoms and a consequent unbinding of atoms from the dendrite. At $1 \mathrm{mM}$ ascorbic acid ( $\mathrm{AA}: \mathrm{Au}^{3+}$ ratios of 6.7) a very interesting intermediate structure has been isolated. Here, numerous spheroidal nanoparticles are formed on the PS nanospheres, with evidence of the onset of surface growth in the form of small "tentacles" of gold protruding radially from them. For even lower concentrations of ascorbic acid (0.25-0.5 mM, corresponding to $\mathrm{AA}: \mathrm{Au}^{3+}$ ratios of 1.7-3.3) surface growth is no longer observed, with gold appearing as surface-bound nanospheroids only. For this concentration range we found that the number of gold particles per core nanosphere could be adjusted simply by varying the amount of PS nanospheres added (Fig. S3 $†$ ). When this amount was very small, the coalescence of some of the surface-bound nanocrystals into elongated islands was promoted (Fig. S3d†). Finally, for the lowest (sub-stoichiometric) ascorbic acid concentrations investigated $(0.05-0.1 \mathrm{mM}$, corresponding to $\mathrm{AA}: \mathrm{Au}^{3+}$ ratios of 0.33-0.67) highly irregular and often elongated gold nanostructures along with many uncoated PS nanospheres are seen.

To further understand the morphological variation of the gold patches we carried out TEM and XRD analysis on samples produced at ascorbic acid concentrations of $100 \mathrm{mM}$ and $0.5 \mathrm{mM}$ (Fig. 3, S4 and S5 $\dagger$ ). Besides confirmation of the highly dendritic structure, Fig. 3a shows that the patches with this structure are extremely thin (estimated thickness of $6 \mathrm{~nm}$ ), further underlining the dominance of diffusion at the PSsolvent interface (rather from the bulk) in their growth. Fig. 3b shows that the surface bound particles which appeared mainly spheroidal in the SEM images of Fig. 1 and 2 are actually rather conical due to the apparently good wetting of the gold for the PS surface. This also demonstrates that, for an ascorbic acid concentration of $0.5 \mathrm{mM}$ at least, these structures are unlikely to have formed in the bulk and attached to the PS nanospheres by heterocoagulation but have in fact heterogeneously nucleated and grown slightly in all accessible dimensions (laterally as well as towards the bulk solvent). Bright- and dark-field images of the same dendritic patchy particle are shown in Fig. $3 \mathrm{c}$ and d respectively. The fairly random pattern of contrast especially seen in the dark field image suggests that the patches are polycrystalline, indicating a diffusion-dominated formation mechanism wherein gold clusters adding to the patch are not able to reorientate to preserve a single crystalline habit. This polycrystallinity is also confirmed by the high resolution TEM 

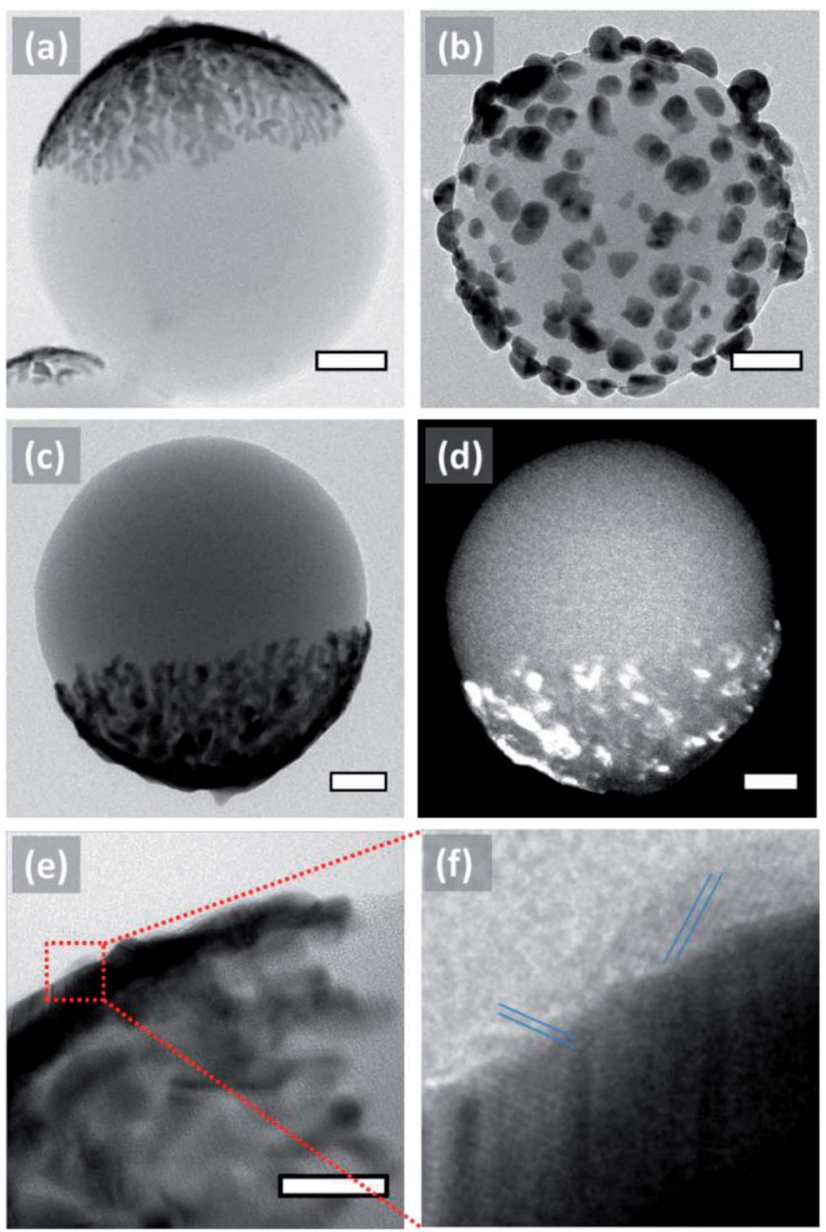

Fig. 3 TEM images of gold nanostructure decorated cores formed by adding equal amounts of chloroauric acid to a mixture of cationic PS nanospheres and ascorbic acid with concentrations of $(a, c-f) 100 \mathrm{mM}$ and (b) $0.5 \mathrm{mM}$. (a-c) Bright field images, (d) dark field image, (e and f) HRTEM images. Scalebars correspond to $50 \mathrm{~nm}$ for $(a-d)$ and $20 \mathrm{~nm}$ for (e).

images (Fig. 3e and f) and by electron diffraction (Fig. S4†). Finally, XRD patterns of samples corresponding to Fig. 3a and $b$ clearly reveal the fcc structure of gold through peaks at 38.2, 44.4, 64.6 and $77.5^{\circ}$ which can be assigned to the (111), (200), (220), and (311) planes respectively (Fig. S5†). ${ }^{43}$

It is interesting to compare some differences in the results between Fig. 1-3 i.e. the result of varying the order of addition of ascorbic and chloroauric acid. When the $\mathrm{AA}: \mathrm{Au}^{3+}$ ratio was 3.3, small dendritic patches were obtained when ascorbic acid was added last (Fig. 1b) but only spheroids were formed when chloroauric acid was added last (Fig. 2 and 3, $0.5 \mathrm{mM}$ ). This difference is most likely due to mixing effects in the case of the earlier experiment - in regions where the local ascorbic acid concentration was high, dendritic patches were able to form. Conversely, when ascorbic acid was added first, an adsorption equilibrium over the whole sample could develop prior to addition of gold. As a result, the structures shown in Fig. 2 and 3 are more narrowly dispersed than in Fig. 1 and the spheroid-todendrite transition appears to occur at a higher ascorbic acid concentration.
We considered whether the patch coverage could be tailored using our approach. One method could be to change the amount of PS core particles while keeping the ascorbic acid and chloroauric acid additions constant. Another could be to increase the amount of gold, keeping the ascorbic acid and PS cores constant. Fig. S6 $\dagger$ demonstrates that both approaches successfully produce gold coatings on the PS nanospheres with a coverage approaching $100 \%$. The fact that coatings approaching complete gold nanoshells could be obtained is consistent with the results of the study on amine multilayer functionalized silica particles by Jang et al. ${ }^{12}$ Our work demonstrates that gold nanoshells could be produced with an even simpler processes, avoiding the need for a functionalization step and making use of just ascorbic acid (i.e. no PVP) to produce the nanoshell.

The strong variation seen in the gold morphology resulting from the concentration of ascorbic acid used is expected to influence the optical properties. Indeed, samples containing dendritic patches had a greenish appearance while those with surface-bound spheroidal patches were purple-coloured. Fig. 4 shows the extinction spectra of gold-PS patchy particles synthesized in the same way as the samples shown in Fig. 2. Due to the strong scattering of polystyrene $(n=1.59)$, features arising from the metal patches are only marginally visible. For the highest concentration of ascorbic acid a clear peak is seen at about $950 \mathrm{~nm}$. This presumably corresponds to the averaged dipole resonance of the large dendritic patches. Compared to our earlier work on (continuous) silver patches this peak is nevertheless rather weak. We believe that this is a result of the significant amount of intrastructural plasmonic coupling leading to broadening of the patch plasmon resonance. Electrodynamic simulations are currently underway to confirm this

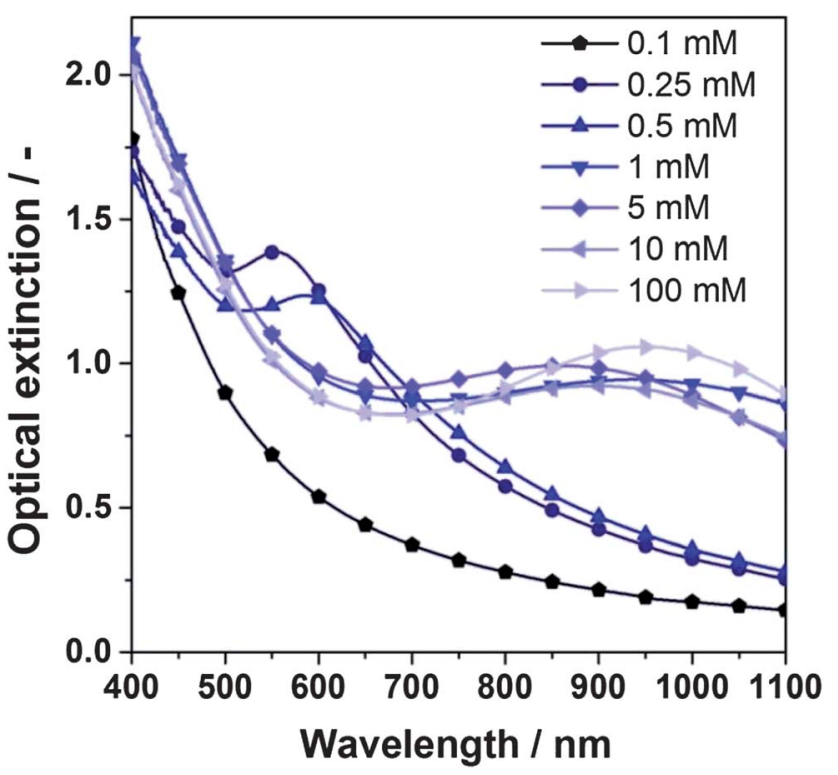

Fig. 4 Optical extinction spectra of gold nanostructure-decorated cores formed by mixing the same amount of cationic PS nanospheres with different concentrations of ascorbic acid followed by addition of the same amount of chloroauric acid. 
effect. These will also show the extent of electromagnetic enhancement close to the dendrites. If this is high, such patchy particles could show great promise as substrates for surface enhanced Raman spectroscopy (SERS), similar to observations with other types of dendritic or rough gold nanoparticles. ${ }^{\mathbf{4 4 , 4 5}}$

Returning to Fig. 4, as the ascorbic acid concentration decreases $(5-10 \mathrm{mM})$ the patch resonance is seen to blue-shift and broaden, most likely the result of the narrowing and fragmentation of the patch. At $1 \mathrm{mM}$ ascorbic acid an unexpected red-shift occurs. This is possibly due to the highly anisotropic morphology (spheroidal nanoparticles with thin lateral protrusions) at this concentration (see Fig. 2). For further reductions in ascorbic acid, a large blue-shift to $600 \mathrm{~nm}(0.5 \mathrm{mM}$ ascorbic acid) is seen. As was observed in Fig. 3c, the gold in this sample is mostly in the form of small conical islands, which could account for the fact that the resonance is red-shifted compared to that expected for a perfect sphere of the same volume. Indeed, when the amount of ascorbic acid is further reduced $(0.25 \mathrm{mM})$, a further blue-shift to about $550 \mathrm{~nm}$ is seen, suggesting the particles become more spherical. Finally, for the lowest ascorbic acid concentration, the incomplete reduction of gold and observed anisotropic nanostructures results in an undetectable optical response and the extinction spectrum is dominated by the polystyrene nanospheres.

\section{Influence of reaction temperature on patchy particle morphology}

Our earlier work regarding silver patch nucleation and growth on silica spheres was strongly influenced by the reaction temperature. ${ }^{21}$ In particular, this parameter was shown to affect both the extent of heterogeneous nucleation and the surfacediffusion mediated growth. In the current work we obtain a high heterogeneous nucleation rate at room temperature resulting in most particles possessing at least one patch. We investigated how the reaction temperature influences the resulting morphology. Fig. 5 shows the results for $100 \mathrm{mM}$ ascorbic acid concentration (AA : $\mathrm{Au}^{3+}$ ratio of 667$)$. In contrast to our earlier work on silver-silica only slight morphological changes can be detected. In particular, coarsening of the dendritic structure can be seen as the temperature increases. This may be a result of the increase of surface diffusion constant of gold, as will also be shown later in our simulations. A more profound effect was observed for lower ascorbic acid concentrations (Fig S7 $\dagger$ ). Here, a distinct spreading of the spheroidal gold nanoparticles over the PS nanospheres surface was observed at the higher temperature. This seems to indicate a slight improvement in surface diffusion but the particle growth is still integration limited.

\section{Discussion of the patch formation mechanism}

The experiments described above show that the one-pot formation of gold patchy particles via the heterogeneous nucleation and growth approach is possible when chloroauric acid is reduced by ascorbic acid in the presence of cationic PS nanospheres. Our original hypothesis was that patch nucleation requires an electrostatic attraction between core particles and
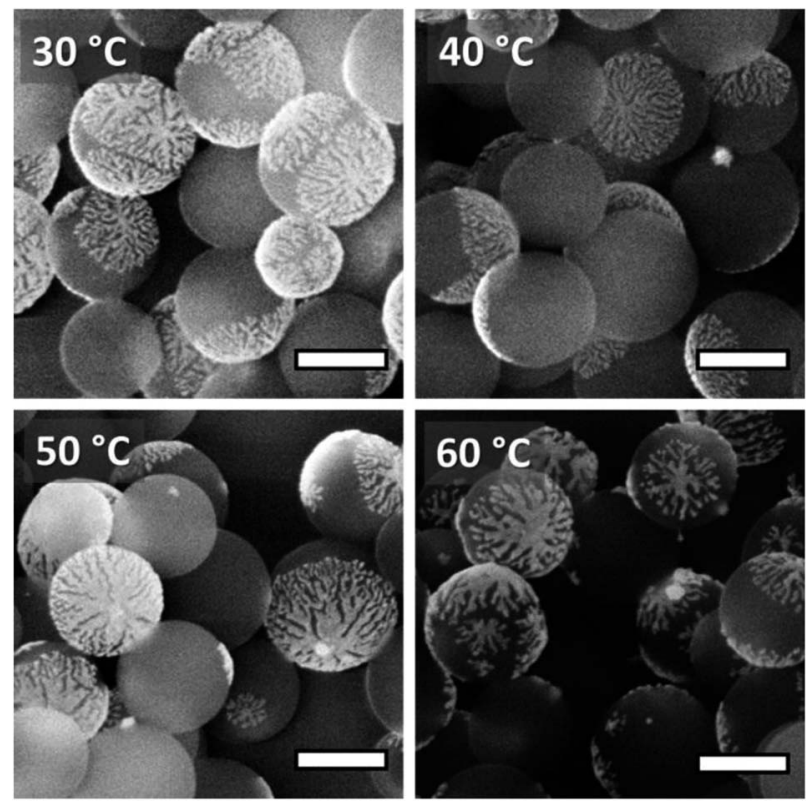

Fig. 5 SEM images of gold nanostructure-decorated cores formed by mixing the same amount of cationic PS nanospheres in $100 \mathrm{mM}$ ascorbic acid followed by addition of the same amount of chloroauric acid. This reaction was carried out at the different temperatures shown. Scalebars correspond to $200 \mathrm{~nm}$.

metal precursor. This would facilitate direct heterogeneous nucleation or promote homogeneous nucleation of gold clusters and rapid adsorption to the surface. Here we have used polystyrene core particles which, by virtue of the azo-initiator employed, possess cationic amidinium surface groups which endow the PS nanospheres with a positive zeta potential over a wide $\mathrm{pH}$ range (see Fig. S $\$$ ). This is expected to provide a strong attraction to the negatively charged chloroauric complex ion and promote heterogeneous nucleation. Given that when the same reaction was carried out in the presence of anionic PS nanospheres, no gold nanostructures attached to the cores were seen (Fig. S9†) it would appear that our hypothesis is correct.

In order to rule out that the chemistry of the polystyrene core particles themselves might have an effect on the patch formation, we carried out the same gold coating procedure on PS and silica core particles which were cationic by virtue of amine groups on their surface (Fig. S10†). In both cases patchy metal coatings with dendritic morphology were formed. While some spheroidal particles were formed on and off the core particle surfaces, it is believed that optimization of the process parameters for these core particles could eliminate this feature. The fact that gold patches can be formed on amine-functionalized PS or silica constitutes further proof of our hypothesis that core particle chemistry is rather irrelevant and that a prerequisite for patch nucleation is that the precursor has an opposite charge to the core particle. This is also consistent with earlier findings that polymer microspheres with different charged functional groups could influence the formation of gold nanospheres of different sizes on the surface. ${ }^{46}$ Differences in the morphologies of the patches in Fig. S10† compared to those formed using amidimium-PS, as used in the rest of this work, can be seen. In 
particular, the dendritic morphology appears to have more separated, "star-like" branches with a wider distribution of lengths than when amidimium-PS was used. These differences presumably arise from the different conditions for adsorption of ascorbic acid and diffusion of gold atoms or clusters on the amine-functionalized surface. Furthermore, in the case silica core nanospheres (Fig. S10b $\dagger$ ) partial separation of the patches from the cores was sometimes observed, indicating that the amine ligand may provide less adhesion for a grown patch. Due to the rapid patch growth reaction $(<1$ minute), the study of differences in patch growth mode for different core functionalization demands more sophisticated in situ analytical techniques. To this end, in our present work we are exploring options such as non-linear optical spectroscopy and in situ TEM.

Having discussed the conditions for patch nucleation, consideration will now be made of parameters which influence the characteristic surface conformal patch growth seen in the present work. In order to obtain patches and not just small spheroidal particles decorating the core nanospheres it is clear that some additional requirements have to be fulfilled. While for the silver-silica system studied previously patch growth was readily achieved over a wide range of conditions, for the gold-PS system considered here surface conformal growth seems to depend critically on the concentration of ascorbic acid. In particular, high excesses of ascorbic acid (at least 40 times the stoichiometric amount) are needed for well-defined patches to be formed. We suggest that enrichment of the PS-nanosphere surface with ascorbic acid promotes the Langmuir-like adsorption of gold, which, once on the surface, maintains lateral mobility. In other words, we suggest the function of ascorbic acid on the surface is to alter the adhesiveness of the surface to gold atoms or clusters. The resulting patches, as we have seen, are highly dendritic in nature and this underlines the relevance of surface diffusion here. Furthermore, for a constant amount of gold precursor added the number of patches per core particle appears to be considerably smaller than the number of surface-bound spheroidal nanoparticles formed at lower ascorbic acid concentrations. This gives the clue that when conditions are perfect for dendritic growth, there is also a reduction in the probability of heterogeneous nucleation. A reason for this might be that at high ascorbic acid concentrations the surface becomes more adhesive for the $\mathrm{AuCl}_{4}{ }^{-}$ion or a reaction intermediate such as $\mathrm{AuCl}_{2}{ }^{-} \cdot{ }^{47}$ Moreover, this interaction appears weak enough to allow the surface mobility of gold. In other words the gold moiety can reside longer at the surface in a non-aggregated state than in the case when the surface is less adhesive, namely at lower concentration of ascorbic acid. Therefore, if the barrier for nucleation is overcome more gold will be present on an adhesive surface to provide material for patch growth. Consequently a nucleus will tend to grow laterally over the surface and competition for gold by this growing patch will result in dendritic growth, as demonstrated by the polycrystallinity of patches observed in our TEM study (Fig. 6). If the surface is non-adhesive to gold then firstly nucleation will more likely be determined by gold arriving directly from the bulk solution via the coulombic interaction referred to above. Secondly, very little lateral growth will be possible due to the lack of surface sites adhesive to gold. Consequently spheroidal surface islands will be formed. In between these two extremes it is expected that there will be a critical concentration of ascorbic acid where the adhesiveness is at an intermediate level so that both appreciable surface diffusion and exchange of gold with the bulk solution can co-exist. Such a situation would be expected to lead to a thinning of the dendrite structure and "pearling", the formation of satellite islands at the edge of the patch. This phenomenon is indeed observed (see Fig. 2, 2.5-5 mM). The appearance of pearls could also indicate some cooperativity between gold atoms that stabilise parts of the structure, an effect that needs to be explored in greater detail.

As suggested above, we hypothesize that an increase in adhesiveness of the PS nanospheres due to the increased concentration of ascorbic acid affects the mobility of gold on the surface of PS. This can be interpreted as a decreasing diffusion constant for gold with increasing the concentration of ascorbic acid and is evidenced by the trend towards finer dendrites (Fig. 2, 5-100 mM). In principle, the density of the dendrite and the thickness of its arms are regulated by the density of gold on the PS particle. However, the dominant effect on the morphology of the dendrite is associated with the DLA parameter $s$. The latter combines two system parameters - the diffusion constant $D$ of gold on the PS surface, and the binding rate of gold atoms to the gold dendrite, regulated by $k_{\text {on }}$ (see eqn (1)). Since there is no indication that the ascorbic acid significantly affects the gold-gold interactions, the main influence on the dendrite morphology must be associated with the change in D. Fig. 6 shows Monte Carlo simulations of gold monolayer domains formed from a constant finite amount of gold and with varying $D$. We firstly found a value of $D$ which leads to a domain morphology similar to that seen in the experiments for the highest concentration of ascorbic acid of $100 \mathrm{mM}$ (i.e. the most gold-adhesive surface). We then found that an increase of $D$ of 1.5 and 6 times was necessary to reproduce the main features of the dendrite morphology at $10 \mathrm{mM}$ and $5 \mathrm{mM}$ respectively (Fig. 6). This shows that reducing the ascorbic acid concentration indeed corresponds to a reduced adhesiveness of the PS surface to gold.

Apart from regulating the morphology of the growth by the ascorbic acid concentration, similar effects are obtained by varying the reaction temperature (Fig. 5). In principle, temperature could affect several aspects of the process. Firstly, the change in entropy of gold in the bulk solution could impact the process of adsorption. Hence the concentration of gold on the surface of the PS particle could be decreased with the increasing temperature. However, since the coverage of the PS colloid does not appear to significantly change with temperature, we assume this effect to be small. It would also be reasonable to assume that increasing temperature could increase the binding rate of gold to the gold dendrite. However, this would imply an increase in the DLA sticking parameter $s$, leading to thinner dendritic morphologies which are not seen in the experiments. However, increased temperature should also increase the lateral diffusion of gold on the PS particle leading 


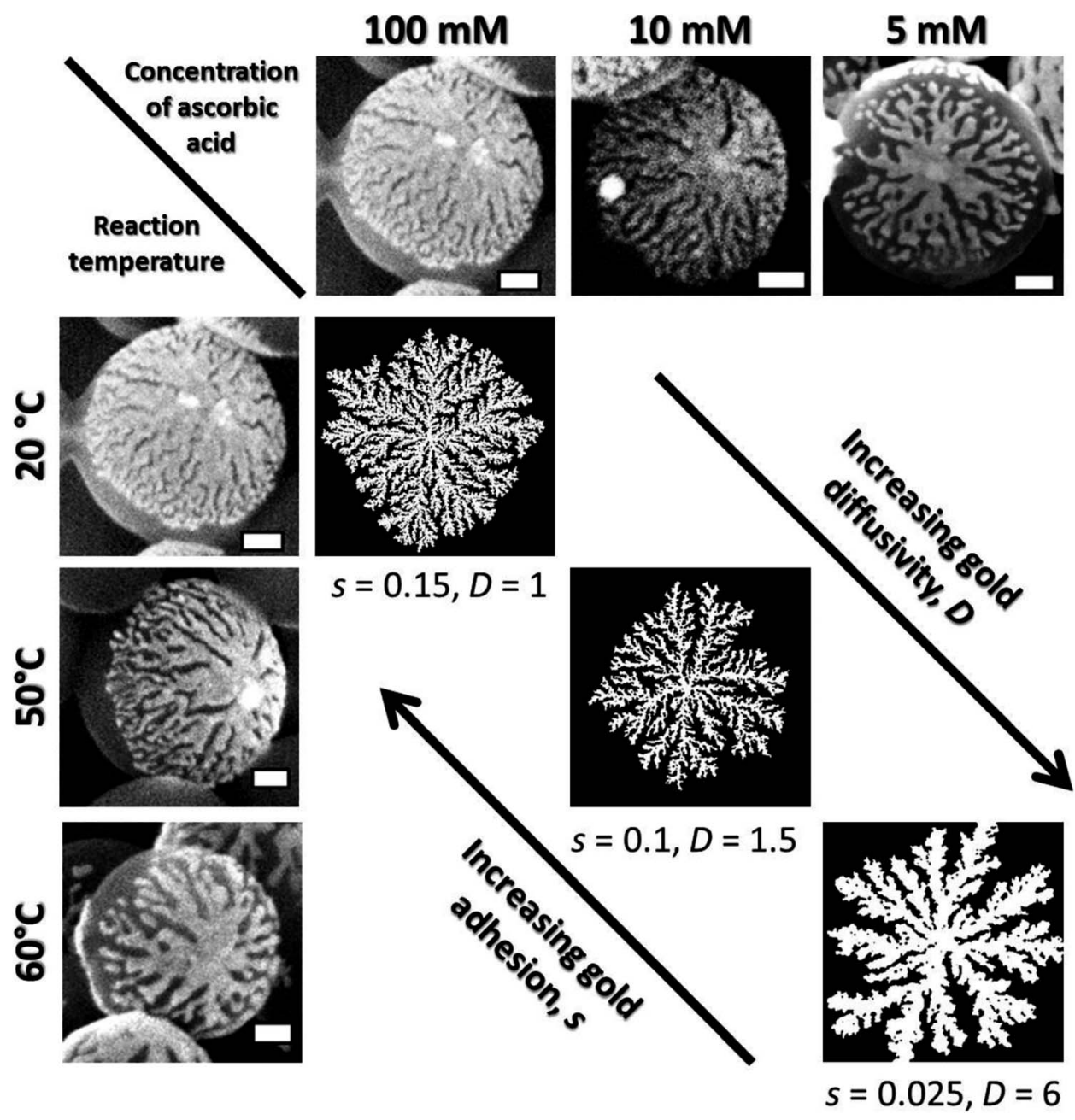

Fig. 6 Comparison of Monte Carlo simulations of gold monolayer domains formed via diffusion limited aggregation (DLA) with actual gold patches produced by the reduction of chloroauric acid by ascorbic acid in the presence of cationic PS nanospheres. The variation of the dendritic morphology, invoked experimentally by the ascorbic acid concentration and reaction temperature is shown to correspond to parameters of the DLA simulations (see text). The density of gold atoms on the simulation grid was 0.3 . Scalebars correspond to $50 \mathrm{~nm}$.

to the observed slight increase in the thickness of the arms (Fig. 5 and 6). Consequently, we conclude that while a number of parameters could be influenced by the temperature, the dominant effect is most likely the change of lateral mobility of gold atoms.

Having shown using simulations that our hypothesis regarding dendritic patch formation is correct, the question remains, what is it about high concentrations of ascorbic acid that make the core particle surface adhesive? Firstly it should be noted that like many "model" nanostructure-producing reactions, the reduction of the chloroauric ion with ascorbic acid is known to be quite complex with a number of possible parallel reactions taking place and intermediates existing. ${ }^{47}$ In particular, the nature of gold reduction depends strongly on the degree of dissociation of the ascorbic acid molecule. As indicated in Table $\mathrm{S} 1, \dagger$ the $\mathrm{pH}$ of the reactions carried out in this work varied between 2.36 (100 $\mathrm{mM}$ ascorbic acid), 3.49 (for $1 \mathrm{mM}$ ascorbic acid) and 8.4 (for $0.05 \mathrm{mM}$ ascorbic acid). With dissociation constants $\mathrm{p} K_{\mathrm{a}, 1}=4.17$ and $\mathrm{p} K_{\mathrm{a}, 2}=11.58$, it is therefore expected that mainly the non-dissociated $\left(\mathrm{H}_{2} \mathrm{Asc}\right)$ and singly deprotonated $\left(\mathrm{HAsc}^{-}\right)$forms participate in the gold reduction reaction. As has been previously noted, the reducing strength of ascorbic acid increases with $\mathrm{pH}$ i.e. with the prevalence of the $\mathrm{HAsc}^{-}$form. ${ }^{47}$ However, in the present work, dendritic gold patch growth occurred preferentially at high ascorbic acid concentrations i.e. at low $\mathrm{pH}$ values, where $\mathrm{H}_{2} \mathrm{Asc}$ is prevalent (see Fig. $\mathrm{S} 11^{\dagger}$ ). Consequently we propose that the main species responsible for the adhesiveness of the PS nanospheres surface for gold is $\mathrm{H}_{2}$ Asc. To test this we carried out the gold coating reaction at three different ascorbic acid concentrations (100 mM, $1 \mathrm{mM}$ and $0.5 \mathrm{mM})$ and for each case carrying out three reactions where we attempted to maintain the $\mathrm{pH}$ at around 2.4, 5.1 and 10.3, where $\mathrm{H}_{2} \mathrm{Asc}, \mathrm{HAsc}^{-}$, $\mathrm{HAsc}^{-}$and $\mathrm{Asc}^{2-}$ are the dominate species, respectively. Additions of hydrochloric acid and sodium hydroxide were used to make the $\mathrm{pH}$ adjustments. The results shown in Fig. 7 indicate that $\mathrm{pH}$ indeed has a strong influence on the gold coating morphology. As before, for high ascorbic acid concentration (100 mM), which anyway leads to a low $\mathrm{pH}$, dendritic patches are formed. For the same ascorbic acid concentration, raising the $\mathrm{pH}$ to 5.3 leads to 

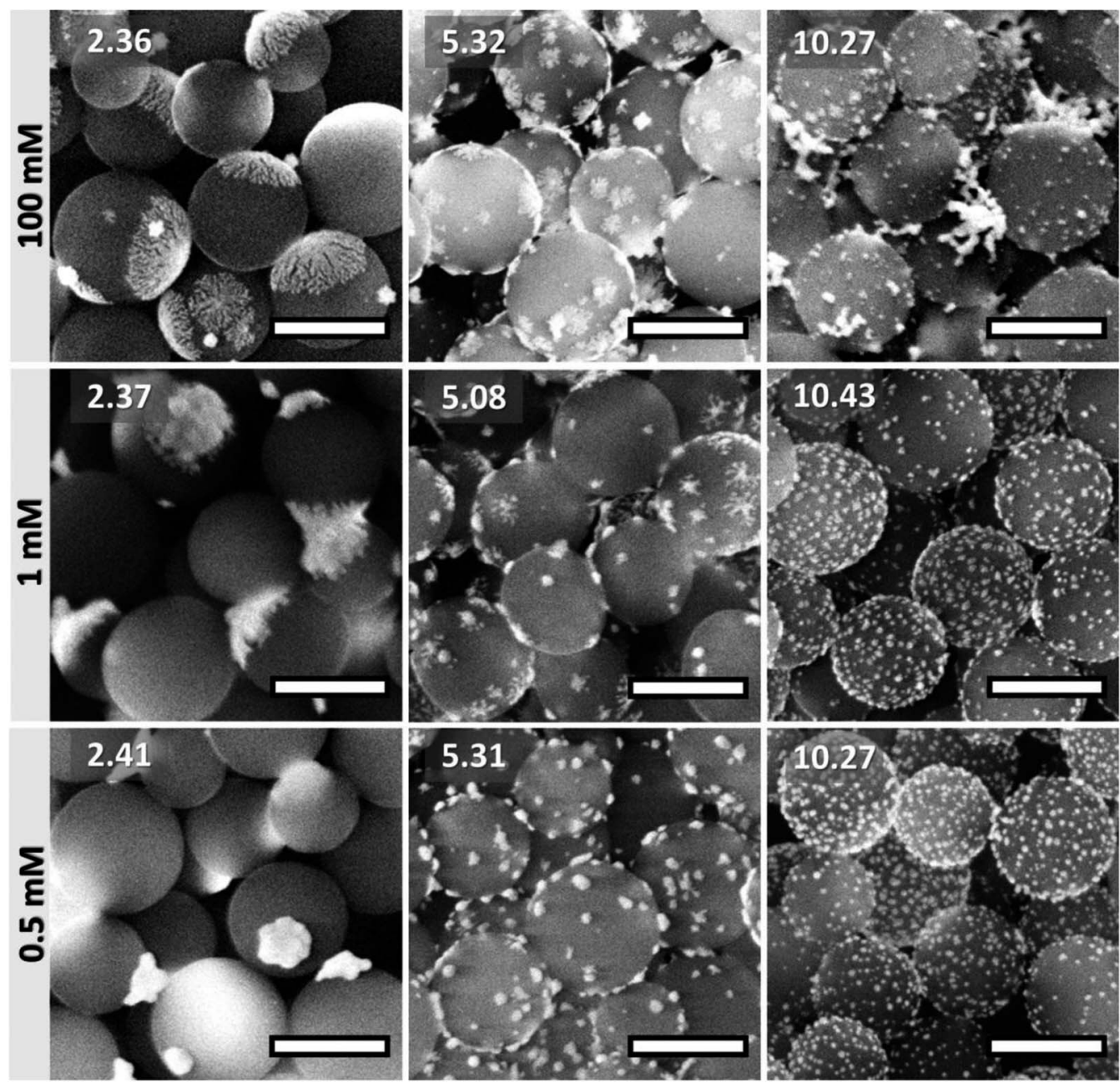

Fig. 7 SEM images of gold nanostructure decorated cores formed by mixing the same amount of cationic PS nanospheres with different concentrations of ascorbic acid (horizontal rows), adjusting the $\mathrm{pH}$ to the value shown on the image label, followed by addition of the same amount of chloroauric acid. Scalebars correspond to $250 \mathrm{~nm}$.

smaller patches with a rather coarse dendritic character in addition to many small nanoparticles which may have resulted from homogeneous nucleation. Finally, at a $\mathrm{pH}$ of 10.27 , many small surface-bound nanoparticles are seen along with aggregates of apparently free nanoparticles. At $1 \mathrm{mM}$ ascorbic acid concentration, deliberate reduction in the naturally occurring $\mathrm{pH}$ leads to the formation of quite thick patches with coarse dendritic edges. Although the yield of these patches is rather low, the surface-conformal growth is unmistakable and the structure is somewhat reminiscent of that obtained with the silver-silica system. ${ }^{21}$ When the $\mathrm{pH}$ is rather increased, small spheroidal particles with dendritic surface protrusions, similar to the structure without pH adjustment (Fig. 2, $1 \mathrm{mM}$ ) are seen. For the highest $\mathrm{pH}$, as with $100 \mathrm{mM}$ ascorbic acid, a large number of small nanoparticles are produced. The trends for coated particles produced at $0.5 \mathrm{mM}$ ascorbic acid concentration are similar to those for $1 \mathrm{mM}$. At reduced $\mathrm{pH}$ evidence for some surface conformal growth is present, although the patch yield is again low and patch thickening has also occurred. At higher $\mathrm{pH}$, mostly spheroidal nanoparticles are again formed.
The study of the $\mathrm{pH}$ influence on the gold-PS nanostructure morphological evolution confirms our earlier suggestion that the non-dissociated form of ascorbic acid $\left(\mathrm{H}_{2} \mathrm{Asc}\right)$ promotes the surface-conformal growth of gold. The specific adsorption of $\mathrm{H}_{2}$ Asc could be expected via strong $\mathrm{N}-\mathrm{H} \cdots \mathrm{O}-\mathrm{C}$ hydrogen bonds between the amidinium groups on the PS and the vinylogous carboxylate group of ascorbic acid. ${ }^{48}$ The surface-adsorbed $\mathrm{H}_{2} \mathrm{Asc}$ would then participate in $\left[\mathrm{AuCl}_{4}\right]^{-}$reduction. How it specifically mediates surface diffusion of gold or its intermediates has yet to be determined and will be the subject of future work. However, it is clear that when the concentration of ascorbic acid decreases a change in the adhesiveness of the surface for gold reduces. Moreover, the $\mathrm{pH}$ increases and the commensurate increase in the amount of $\mathrm{HAsc}^{-}$leads to an increase in the rate of reduction. This would explain the preference for a larger number of spheroidal gold nanoparticles to be nucleated on the PS nanospheres at lower ascorbic acid concentrations (see Fig. $21 \mathrm{mM}$ ascorbic acid and lower), and is possibly also due to the fact that $\left[\mathrm{AuCl}_{4}\right]^{-}$will undergo partial hydrolysis to $\left[\mathrm{Au}(\mathrm{OH})_{x} \mathrm{Cl}_{4-x}\right]^{-} \cdot{ }^{47}$ 


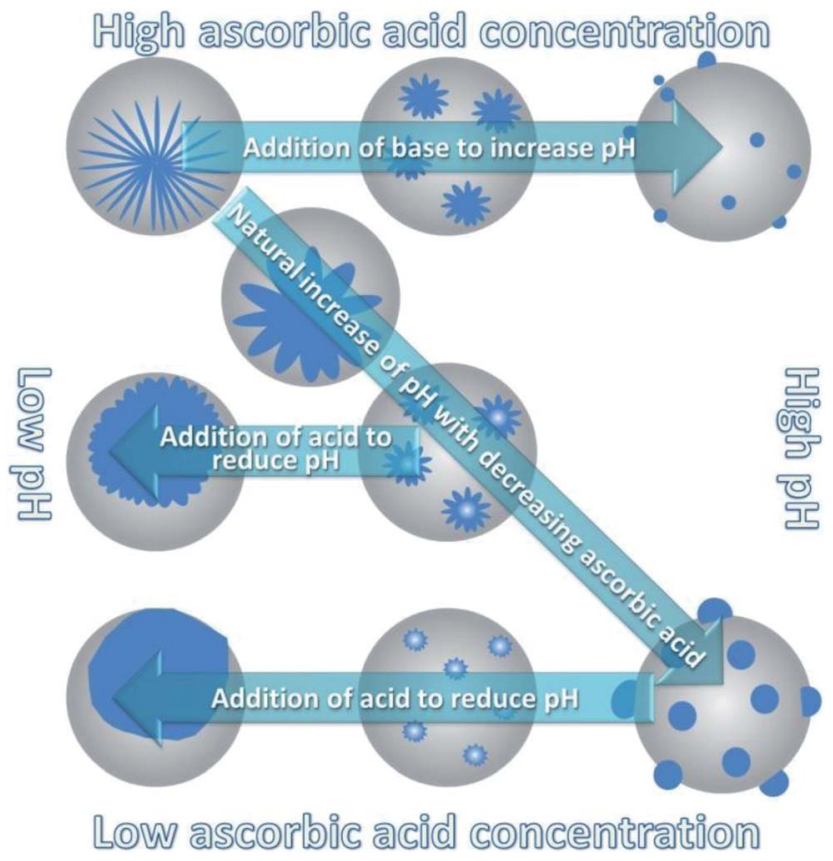

Scheme 1 Schematic representation of the influence of reaction parameters on the morphological variation of gold nanostructures on cationic PS nanospheres.

Since the moiety which we propose makes the PS more adhesive for gold $\left(\mathrm{H}_{2} \mathrm{Asc}\right)$ is highly $\mathrm{pH}$ sensitive and the ascorbic acid concentration itself determines the $\mathrm{pH}$, surface conformal growth could also be activated at lower concentrations of ascorbic acid by adjustment of the $\mathrm{pH}$. Scheme 1 summarizes the key reaction parameters which influence the morphological evolution of gold coatings on cationic PS nanospheres as determined by this study.

\section{Conclusions}

A simple and environmentally-friendly method of preparing gold patches on cationic polystyrene nanospheres in aqueous solution has been described. The strategy was based on the novel heterogeneous nucleation and growth approach whereby chloroauric acid was reduced by ascorbic acid in the presence of the core particles. The heterogeneous nucleation of patches was shown to depend mainly on the electrostatic interaction between core and metal precursor. Moreover, the lateral surface growth of gold patches was highly dependent on the ascorbic acid concentration, with an excess of at least 40 times the stoichiometric amount required to produce large thin gold patches on the nanospheres. The patches had a highly dendritic morphology and the dependence of this on reactant concentration, $\mathrm{pH}$ and temperature were studied. Furthermore, Monte Carlo simulations assisted with the development of a model for the surface conformal growth. In the latter it is believed that the non-dissociated form of ascorbic acid is responsible for adsorbing to the core particles and providing an adhesive surface for gold. It was shown that non-dendritic patches could be formed if a lower concentration of ascorbic acid was used and the $\mathrm{pH}$ deliberately reduced. These conditions resulted in a less adhesive surface for gold but avoided the fast reduction which leads to the nucleation and growth of small spheroidal nanoparticles bound to the polystyrene surface. The patchy particles produced in this work were shown to possess morphology-dependent optical properties, a result of the plasmonic resonances of the highly anisotropic gold coatings. These properties, along with the surface anisotropy are expected to make the patchy particles produced according to the novel method interesting candidates for up-coming studies of selfand directed-organization, self-propelling nanoswimmers, particulate surfactants and plasmonic sensors.

\section{Acknowledgements}

The authors gratefully acknowledge the support of the German Research Foundation (DFG), which, within in the framework of its 'Excellence Initiative', supports the Cluster of Excellence 'Engineering of Advanced Materials' at FAU Erlangen-Nürnberg. We kindly acknowledge Prof. Udo Seifert for helpful discussions.

\section{Notes and references}

1 G. Loget and A. Kuhn, J. Mater. Chem., 2012, 22, 15457.

2 J. Du and R. K. O'Reilly, Chem. Soc. Rev., 2011, 40, 2402.

3 E. Bianchi, R. Blaak and C. N. Likos, Phys. Chem. Chem. Phys., 2011, 13, 6397.

4 A. B. Pawar and I. Kretzschmar, Macromol. Rapid Commun., 2010, 31, 150.

5 S. Jiang, Q. Chen, M. Tripathy, E. Luijten, K. S. Schweizer and S. Granick, Adv. Mater., 2010, 22, 1060.

6 A. Walther and A. H. E. Müller, Chem. Rev., 2013, 113, 5194.

7 J. Yan, M. Bloom, S. C. Bae, E. Luijten and S. Granick, Nature, 2012, 491, 530.

8 A. Walther, M. Hoffmann and A. H. E. Müller, Angew. Chem., Int. Ed., 2008, 47, 711.

9 D. Kagan, R. Laocharoensuk, M. Zimmerman, C. Clawson, S. Balasubramanian, D. Kang, D. Bishop, S. Sattayasamitsathit, L. Zhang and J. Wang, Small, 2010, 6, 2741.

10 H. Takei and N. Shimizu, Langmuir, 1997, 13, 1865.

11 D. Rodriguez-Fernandez and L. M. Liz-Marzan, Part. Part. Syst. Charact., 2013, 30, 46.

12 S. G. Jang, S. H. Kim, S. Y. Lee, W. C. Jeong and S. M. Yang, J. Colloid Interface Sci., 2010, 350, 387.

13 C. Bae, J. Moon, H. Shin, J. Kim and M. M. Sung, J. Am. Chem. Soc., 2007, 129, 14232.

14 X. Fu, J. Liu, H. Yang, J. Sun, X. Li, X. Zhang and Y. Jia, Mater. Chem. Phys., 2011, 130, 334.

15 Y. Xia, Y. Yang, J. Zheng, W. Huang and Z. Li, RSC Adv., 2012, 2, 5284 .

16 V. N. Paunov and O. J. Cayre, Adv. Mater., 2004, 16, 788.

17 C. Xu, B. Wang and S. Sun, J. Am. Chem. Soc., 2009, 131, 4216.

18 S. Pradhan, D. Ghosh and S. Chen, ACS Appl. Mater. Interface, 2009, 1, 2060. 
19 C. Casagrande and M. Veyssie, C. R. Acad. Sci., Ser. II: Mec., Phys., Chim., Sci. Terre Univers, 1988, 306, 1423.

20 L. Hong, S. Jiang and S. Granick, Langmuir, 2006, 22, 9495.

21 H. Bao, R. N. Klupp Taylor and W. Peukert, Adv. Mater., 2011, 23, 2644.

22 H. Bao, B. Butz, Z. Zhou, E. Spiecker, M. Hartmann and R. N. Klupp Taylor, Langmuir, 2012, 28, 8971.

23 R. L. Oliveira, D. Zanchet, P. K. Kiyohara and L. M. Rossi, Chem.-Eur. J., 2011, 17, 4626.

24 A. Sokoutchaev, J. T. James, S. C. Koene, S. Pathak, G. K. S. Prakash and M. E. Thompson, Chem. Mater., 1999, 11, 2389.

25 J. H. Lee, M. A. Mahmoud, V. Sitterle, J. Sitterle and J. C. Meredith, J. Am. Chem. Soc., 2009, 131, 5048.

26 W. L. Shi, Y. Sahoo, M. T. Swihart and P. N. Prasad, Langmuir, 2005, 21, 1610.

27 Z. J. Liang, A. S. Susha and F. Caruso, Adv. Mater., 2002, 14, 1160.

28 J. Lee, M. A. Mahmoud, V. B. Sitterle, J. J. Sitterle and J. C. Meredith, Chem. Mater., 2009, 21, 5654.

29 J. Zhang, J. Liu, S. Wang, P. Zhan, Z. Wang and N. Ming, Adv. Funct. Mater., 2004, 14, 1089.

30 A. Dong, Y. Wang, Y. Tang, N. Ren, W. Yang and Z. Gao, Chem. Commun., 2002, 350.

31 J. Gong, X. Zu, W. Mu and Y. Deng, Colloid Polym. Sci., 2013, 291, 239.

32 Y. Li, Z. Huang, Y. Wu, C. Yang, Y. Gao and Z. Wang, Colloids Surf., A, 2012, 407, 71.

33 Y. Li, Y. Pan, L. Zhu, Z. Wang, D. Su and G. Xu, Macrmol. Rapid Commun., 2011, 32, 1741.

34 K. Y. van Berkel, G. T. Russell and R. G. Gilbert, Macromolecules, 2003, 36, 3921.
35 A.-L. Barabási, Fractal concepts in surface growth, Cambridge University Press, 1995; P. Meakin, Physica D, 1995, 86, 104; T. A. Witten Jr and L. M. Sander, Phys. Rev. Lett., 1981, 47, 1400.

36 P. Meakin, Phys. Rev. A, 1983, 27, 1495.

37 R. F. Voss and M. Tomkiewicz, J. Electrochem. Soc., 1985, 132, 371.

38 J. Choi, D. Crowdy and M. Z. Bazant, Europhys. Lett., 2010, 91, 46005.

39 A. M. Bode, L. Cunningham and R. C. Rose, Clin. Chem., 1990, 36, 1807.

40 G. Sharma and M. Ballauff, Macromol. Rapid Commun., 2004, $25,547$.

41 M. Schrinner, S. Proch, Y. Mei, R. Kempe, N. Miyajima and M. Ballauff, Adv. Mater., 2008, 20, 1928.

42 J. Lee, D. O. Kim, G. Song, Y. Lee, S. Jung and J. Nam, Macromol. Rapid Commun., 2007, 28, 634.

43 Y. Sun and Y. Xia, Science, 2002, 298, 2176.

44 X. Tang, P. Jiang, G. Ge, M. Tsuji, S. Xie and Y. Guo, Langmuir, 2008, 24, 1763.

45 P. Aldeanueva-Potel, E. Carbó-Argibay, N. Pazos-Pérez, S. Barbosa, I. Pastoriza-Santos, R. A. Alvarez-Puebla and L. M. Liz-Marzán, ChemPhysChem, 2012, 13, 2561.

46 W. Liu, X. Yang and L. Xie, J. Colloid Interface Sci., 2007, 313, 494.

47 M. Luty-Blocho, K. Paclawski, M. Wojnicki and K. Fitzer, Inorg. Chim. Acta, 2013, 395, 189.

48 I. Kuzmenko, M. Kindermann, K. Kjaer, P. B. Howes, J. AlsNielsen, R. Granek, G. v. Kiedrowski, L. Leiserowitz and M. Lahav, J. Am. Chem. Soc., 2001, 123, 3771. 